\title{
On a nonlinear second order periodic boundary value problem with Carathéodory functions
}

\author{
by Wenje GaO and Junyu Wang (Changchun)
}

\begin{abstract}
The periodic boundary value problem $u^{\prime \prime}(t)=f\left(t, u(t), u^{\prime}(t)\right)$ with $u(0)=$ $u(2 \pi)$ and $u^{\prime}(0)=u^{\prime}(2 \pi)$ is studied using the generalized method of upper and lower solutions, where $f$ is a Carathéodory function satisfying a Nagumo condition. The existence of solutions is obtained under suitable conditions on $f$. The results improve and generalize the work of M.-X. Wang et al. [5].
\end{abstract}

1. Introduction. In recent years, a number of authors have studied the following periodic boundary value problem of second order:

$$
\begin{aligned}
& -u^{\prime \prime}(t)=f\left(t, u(t), u^{\prime}(t)\right), \\
& u(0)=u(2 \pi), \quad u^{\prime}(0)=u^{\prime}(2 \pi) .
\end{aligned}
$$

People mainly studied the problem for $f$ continuous with respect to its variables (see $[1-5]$ and the references therein).

In [5], M.-X. Wang, A. Cabada and J. Nieto studied (1.1) when $f$ is a Carathéodory function, using a generalized upper and lower solution method. Also, they developed a monotone iterative technique for finding minimal and maximal solutions.

In this paper, we use a modified version of the method of [5] to study the existence of solutions to problem (1.1) and develop a monotone iterative technique for finding the minimal and maximal solutions. Our method substantially modifies that of [5] and part of our results improve and generalize the results obtained in [5]. With our method, it is possible to extend the result to a more general form.

For completeness, we include some of the results of [5] with their (or modified) proofs. We use the same definitions and notations as in [5]. We

1991 Mathematics Subject Classification: Primary 34B15, 34B10, 34C25.

Key words and phrases: two-point boundary value problems, upper and lower solutions, Nagumo condition, existence, Carathéodory functions.

The authors are partially supported by NNSF of China. 
write $I=[0,2 \pi]$ and denote by $W^{2,1}(I)$ the set of functions defined in $I$ with integrable second derivatives and define the sector $[\alpha, \beta]$ as the set $[\alpha, \beta]=\left\{u \in W^{2,1}(I): \alpha(t) \leq u(t) \leq \beta(t)\right.$ for $\left.t \in I=[0,2 \pi]\right\}$.

We call a function $f: I \times \mathbb{R}^{2} \rightarrow \mathbb{R}$ a Carathéodory function if the following conditions are satisfied:

(1) for almost all $t \in I$, the function $\mathbb{R}^{2} \ni(u, v) \rightarrow f(t, u, v) \in \mathbb{R}$ is continuous;

(2) for every $(u, v) \in \mathbb{R}^{2}$, the function $I \ni t \rightarrow f(t, u, v)$ is measurable;

(3) for every $M>0$, there exists a real-valued function $\phi(t)=\phi_{M}(t) \in$ $L^{1}(I)$ such that

$$
|f(t, u, v)| \leq \phi(t)
$$

for a.e. $t \in I$ and every $(u, v) \in \mathbb{R}^{2}$ satisfying $|u| \leq M$ and $|v| \leq M$. and

We call a function $\alpha: I \rightarrow \mathbb{R}$ a lower solution of (1.1) if $\alpha \in W^{2,1}(I)$

$$
\begin{aligned}
& -\alpha^{\prime \prime}(t) \leq f\left(t, \alpha(t), \alpha^{\prime}(t)\right) \quad \text { for a.e. } t \in I, \\
& \alpha(0)=\alpha(2 \pi), \quad \alpha^{\prime}(0) \geq \alpha^{\prime}(2 \pi) .
\end{aligned}
$$
and

$$
\begin{aligned}
& -\beta^{\prime \prime}(t) \geq f\left(t, \beta(t), \beta^{\prime}(t)\right) \quad \text { for a.e. } t \in I, \\
& \beta(0)=\beta(2 \pi), \quad \beta^{\prime}(0) \leq \beta^{\prime}(2 \pi) .
\end{aligned}
$$

The following hypothesis is adopted:

(H1) The nonlinear function $f$ satisfies the Nagumo condition on the set

$$
\Omega:=\{(t, u, v): 0 \leq t \leq 2 \pi, \alpha(t) \leq u \leq \beta(t), v \in \mathbb{R}\},
$$

i.e. there exist a real-valued function $h(t) \in L^{\sigma}(I), 1 \leq \sigma \leq \infty$, and a continuous function $g(v): \mathbb{R}^{+} \rightarrow \mathbb{R}^{+}$such that

$$
|f(t, u, v)| \leq h(t) g(|v|) \quad \text { on } \Omega,
$$

and

$$
\int_{0}^{\infty} \frac{u^{(\sigma-1) / \sigma}}{g(u)} d u>\varrho^{(\sigma-1) / \sigma}\|h\|_{\sigma}
$$

where

$$
\varrho=\max _{t \in I} \beta(t)-\min _{t \in I} \alpha(t)
$$

and

$$
\|h\|_{\sigma}= \begin{cases}\left(\int_{0}^{2 \pi}(h(t))^{\sigma} d t\right)^{1 / \sigma} & \text { for } \sigma \in(0, \infty) \\ \sup _{t \in[0,2 \pi]}|h(t)| & \text { for } \sigma=\infty\end{cases}
$$


Remark. In our paper, the Nagumo condition is defined in a slightly different way than in [5]. Our definition includes theirs as a special case. In fact, it is easy to see that under their definition the combination of their Carathéodory condition and the Nagumo condition implies that the function $h(t)$ in their paper must be bounded when $u \in[\alpha, \beta]$ and $v$ is in a bounded set.

2. Existence of solutions. For any $u \in X=C^{1}(I)$, we define

$$
p(t, u)= \begin{cases}\alpha(t), & u(t)<\alpha(t), \\ u(t), & \alpha(t) \leq u(t) \leq \beta(t), \\ \beta(t), & u(t)>\beta(t) .\end{cases}
$$

The following lemma is Lemma 2 of [5]:

Lemma 1. For $u \in X$, the following two properties hold:

(1) $\frac{d}{d t} p(t, u(t))$ exists for a.e. $t \in I$.

(2) If $u, u_{m} \in X$ and $u_{m} \rightarrow u$ in $X$, then

$$
\frac{d}{d t} p\left(t, u_{m}(t)\right) \rightarrow \frac{d}{d t} p(t, u(t)) \quad \text { for a.e. } t \in I .
$$

Proof. Note that $p(t, u)=[u-\alpha]^{-}-[u-\beta]^{+}+u$, where $u^{+}(t)=$ $\max \{u(t), 0\}$ and $u^{-}(t)=\max \{-u(t), 0\}$. The first assertion is obvious since $u^{+}$and $u^{-}$are absolutely continuous for $u \in X$. To prove the second, we only have to show that if $u, u_{m} \in X$ and $u_{m} \rightarrow u$ in $X$, then

$$
\lim _{m \rightarrow \infty} \frac{d}{d t} p\left(t, u_{m}^{ \pm}\right)(t)=\frac{d}{d t} p\left(t, u^{ \pm}\right)(t) \quad \text { for a.e. } t \in I \text {. }
$$

We only need to check the limit at a point $t_{0} \in I$ where $\frac{d}{d t} u_{m}^{+}$and $\frac{d}{d t} u^{+}$ exist for all $m=1,2, \ldots$.

If $u\left(t_{0}\right)>0$, then $u\left(t_{0}\right)=u^{+}\left(t_{0}\right)>0$. Therefore $\frac{d}{d t} u^{+}\left(t_{0}\right)=\frac{d}{d t} u\left(t_{0}\right)$ and there exists an $M>0$ such that $u_{m}\left(t_{0}\right)=u_{m}^{+}\left(t_{0}\right)>0$ for all $m>M$. Thus

$$
\frac{d}{d t} u_{m}^{+}\left(t_{0}\right)=\frac{d}{d t} u_{m}\left(t_{0}\right) \rightarrow \frac{d}{d t} u\left(t_{0}\right) .
$$

If $u\left(t_{0}\right)<0$, then $\frac{d}{d t} u^{+}\left(t_{0}\right)=0$ and there exists an $M>0$ such that $u_{m}^{+}(t)=0$ on $\left(t_{0}-\delta_{m}, t_{0}+\delta_{m}\right)$ for some $\delta_{m}>0$ for all $m>M$. Therefore $\frac{d}{d t} u^{+}\left(t_{0}\right)=0=\lim \frac{d}{d t} u_{m}^{+}\left(t_{0}\right)$.

If $u\left(t_{0}\right)=0$, then $u^{+}\left(t_{0}\right)=0$. Since $\frac{d}{d t} u^{+}\left(t_{0}\right)$ exists, we have $\frac{d}{d t} u^{+}\left(t_{0}\right)$ $=0$. It is obvious that $\frac{d}{d t} u\left(t_{0}\right)=0$. Then

$$
\left|\frac{d}{d t} u_{m}^{+}\left(t_{0}\right)\right| \leq\left|\frac{d}{d t} u_{m}\left(t_{0}\right)\right| \rightarrow\left|\frac{d}{d t} u\left(t_{0}\right)\right|=0=\frac{d}{d t} u^{+}\left(t_{0}\right) .
$$

The proof for $u^{-}$is similar and thus the proof of Lemma 1 is complete. 

lem:

To study the problem (1.1), we first consider the following modified prob-

$$
\begin{aligned}
& -u^{\prime \prime}+u=f^{*}\left(t, p(t, u), \frac{d p(t, u)}{d t}\right)+p(t, u), \\
& u(0)=u(2 \pi), \quad u^{\prime}(0)=u^{\prime}(2 \pi)
\end{aligned}
$$

where

$$
f^{*}(t, u, v)= \begin{cases}f(t, u, N) & \text { if } v>N \\ f(t, u, v) & \text { if }|v| \leq N \\ f(t, u,-N) & \text { if } v<-N\end{cases}
$$

We may choose $N$ so large that

$$
N>\max \left\{\sup _{t \in I}\left|\beta^{\prime}(t)\right|, \sup _{t \in I}\left|\alpha^{\prime}(t)\right|\right\},
$$

and

$$
\int_{0}^{N} \frac{u^{(\sigma-1) / \sigma}}{g(u)} d u>\varrho^{(\sigma-1) / \sigma}\|h\|_{\sigma}
$$

(H1) assures the existence of such an $N$.

For each $q \in X$, we define

$$
\xi(q)(t)=\xi(t)=f^{*}\left(t, p(t, q(t)), \frac{d p(t, q(t))}{d t}\right)+p(t, q(t)),
$$

and consider the problem

$$
\begin{aligned}
& -u^{\prime \prime}+u=\xi(t), \\
& u(0)=u(2 \pi), \quad u^{\prime}(0)=u^{\prime}(2 \pi) .
\end{aligned}
$$

It is obvious that the solution of (2.3) can be written in the form

$$
u(t)=C_{1} e^{t}+C_{2} e^{-t}-\frac{e^{t}}{2} \int_{0}^{t} \xi(s) e^{-s} d s+\frac{e^{-t}}{2} \int_{0}^{t} \xi(s) e^{s} d s
$$

where

$$
\begin{aligned}
C_{1} & =\frac{1}{2\left(e^{2 \pi}-1\right)} \int_{0}^{2 \pi} \xi(s) e^{2 \pi-s} d s, \\
C_{2} & =\frac{1}{2\left(e^{2 \pi}-1\right)} \int_{0}^{2 \pi} \xi(s) e^{s} d s .
\end{aligned}
$$

Lemma 1 obviously implies that $\xi(t)$ is measurable and

$$
\left|f^{*}\left(t, p(t, q(t)), \frac{d p(t, q(t))}{d t}\right)\right| \leq \phi(t) \in L^{1}(I) .
$$


Hence, $\xi \in L^{1}(I)$. Differentiating (2.4) with respect to $t$, we obtain

$$
u^{\prime}(t)=C_{1} e^{t}-C_{2} e^{-t}-\frac{e^{t}}{2} \int_{0}^{t} \xi(s) e^{-s} d s+\frac{e^{-t}}{2} \int_{0}^{t} \xi(s) e^{s} d s,
$$

which is obviously continuous. Therefore, the solution of (2.3) is in $X$ for any $q \in X$.

Define the operator $T: X \rightarrow X$ by $T[q]=u$, with $u$ defined by (2.4). As in [5], we have the following

LemMA 2. $T: X \rightarrow X$ is compact.

Proof. Suppose that $\left\{q_{m}\right\} \subset X$ is such that $q_{m} \rightarrow q$ in $X$. By Lemma 1 , $p\left(t, q_{m}\right) \rightarrow p(t, q)$ and $\frac{d}{d t} p\left(t, q_{m}\right) \rightarrow \frac{d}{d t} p(t, q)$ a.e. Then the properties of $f$ and the Lebesgue dominated convergence theorem imply that

$$
\lim _{m \rightarrow \infty} \int_{0}^{t} \xi_{m}(s) e^{ \pm s} d s=\int_{0}^{t} \xi(s) e^{ \pm s} d s
$$

where

$$
\xi_{m}=f^{*}\left(t, p\left(t, q_{m}(t)\right), \frac{d p\left(t, q_{m}(t)\right)}{d t}\right)+p\left(t, q_{m}(t)\right) .
$$

Therefore, (2.4) and (2.5) show that $T\left[q_{m}\right] \rightarrow T[q]$ in $X$, i.e., $T$ is continuous from $X$ to $X$.

Now, we only have to show that $T$ maps every bounded sequence in $X$ to a compact sequence in $X$. Since $\left|\xi_{m}(s)\right| \leq h(s) g(N)+|\alpha(s)|+|\beta(s)| \in L^{1}(I)$, the sequence $\int_{0}^{t} \xi_{m}(s) e^{ \pm s} d s$ is equicontinuous, and so are $T\left[q_{m}\right]$ and $\frac{d}{d t} T\left[q_{m}\right]$. The Arzelà-Ascoli Theorem implies that $T$ is compact.

Lemma 3. Let $u \in W^{2,1}(I)$ with $u^{\prime \prime}(t) \geq M(t) u(t)$ for a.e. $t \in I, u(0)=$ $u(2 \pi)$ and $u^{\prime}(0) \geq u^{\prime}(2 \pi)$, where $M(t) \in L^{1}(I)$ and $M(t)>0$. Then $u(t) \leq 0$ fro every $t \in I$.

Proof. Set $G=\{t \in I: u(t)>0\}$. Then $u^{\prime \prime}(t)>0$ on $G$. If $G \supset(0,2 \pi)$, then

$$
u^{\prime}(2 \pi) \geq u^{\prime}(0)+\int_{0}^{2 \pi} M(t) u(t) d t>u^{\prime}(0),
$$

which is impossible. Hence, there exists at least one $\tau \in I$ with $u(\tau) \leq 0$. If $u(0)>0$, then there exist $0<s_{1} \leq s_{2}<2 \pi$ with $u\left(s_{1}\right)=u\left(s_{2}\right)=0$ and $u(s)>0$ for $s \in J=\left[0, s_{1}\right) \cup\left(s_{2}, 2 \pi\right]$. Therefore, $u^{\prime}$ is nondecreasing in $\left[0, s_{1}\right)$ and $\left(s_{2}, 2 \pi\right]$. But

$$
u^{\prime}(0)<u^{\prime}\left(s_{1}\right) \leq 0 \leq u^{\prime}\left(s_{2}\right)<u^{\prime}(2 \pi),
$$

a contradiction. 
If $u(0) \leq 0$ and $\max \{u(s): s \in I\}=u\left(t_{0}\right)>0$ then there exist $t_{1}, t_{2} \in$ $(0,2 \pi)$ such that $t_{1}<t_{0}<t_{2}, u\left(t_{1}\right)=u\left(t_{2}\right)=0$ and $u(s)>0$ for $s \in\left(t_{1}, t_{2}\right)$. This implies that $u$ is convex on $\left[t_{1}, t_{2}\right]$ and hence $u(t) \leq 0$ on $\left[t_{1}, t_{2}\right]$, which is impossible. Therefore $u(s) \leq 0$, and the proof is complete.

Now, we are ready to show the existence of solutions for the problem (1.1). We have

THeOREM 1. Suppose that $\alpha(t), \beta(t)$ are lower and upper solutions of problem (1.1) respectively, and $\alpha(t) \leq \beta(t)$ on I. If ( $\mathrm{H} 1)$ holds, then there exists a solution $u$ of (1.1) such that $u \in[\alpha, \beta]$.

Pr o of. We first consider the operator $T$ defined as above. It is easy to verify from (2.4) and (2.5) that $T$ maps $X$ to a bounded subset of $X$. Hence, by the compactness of the operator and the Schauder fixed point principle, we know that there exists a function $u \in X$ such that $u=T[u]$. Such a $u$ is obviously a solution of problem (2.1), therefore, it suffices to show that $u \in[\alpha, \beta]$ and $\left|u^{\prime}\right| \leq N$.

We first show that $u \in[\alpha, \beta]$. Indeed, if $u>\beta$ on $I$, then $p(t, u)=\beta$. Therefore,

$$
-u^{\prime \prime}+u=f\left(t, \beta, \beta^{\prime}\right) \leq-\beta^{\prime \prime}+\beta
$$

by the definition of $f^{*}$ and the choice of $N$. Lemma 3 then implies that $u \leq \beta$ on $I$, a contradiction. Therefore there must be a point $s \in I$ with $u(s) \leq \beta(s)$. If $u(0) \leq \beta(0)$ and there exists $s_{1} \in(0,2 \pi)$ with $u\left(s_{1}\right)>\beta\left(s_{1}\right)$, then by the continuity of $u$, we know that there would be $t_{1}<s_{1}<t_{2}$ in $(0,2 \pi)$ such that $u>\beta$ on $\left(t_{1}, t_{2}\right)$ with $(u-\beta)\left(t_{1}\right)=(u-\beta)\left(t_{2}\right)=0$. Then (2.6) holds in the interval $\left(t_{1}, t_{2}\right)$. This and the boundary conditions imply that $u \leq \beta$ on $\left(t_{1}, t_{2}\right)$, which is again a contradiction.

If $u(0)>\beta(0)$, then there exist $t_{1}<t_{2}$ in $I$ such that $u>\beta$ on $\left[0, t_{1}\right) \cup$ $\left(t_{2}, 2 \pi\right]$ with $(u-\beta)\left(t_{1}\right)=(u-\beta)\left(t_{2}\right)=0$ and hence $(u-\beta)^{\prime}\left(t_{1}\right) \leq 0$ and $(u-\beta)^{\prime}\left(t_{2}\right) \geq 0$. In both intervals, $(u-\beta)^{\prime \prime} \geq u-\beta>0$. Hence, $(u-\beta)^{\prime}$ is increasing, which implies that $(u-\beta)^{\prime}(0)<(u-\beta)^{\prime}\left(t_{1}\right) \leq 0$ and $(u-\beta)^{\prime}(2 \pi)>(u-\beta)^{\prime}\left(t_{2}\right) \geq 0$, contrary to the boundary conditions.

To sum up, we know that $u \leq \beta$ on $I$. Analogously we can prove that $u \geq \alpha$.

All that remains to be proved is that $\left|u^{\prime}\right| \leq N$.

The mean value theorem asserts that there exists a point $t_{0} \in I$ such that $u^{\prime}\left(t_{0}\right)=0$. Assume that $\left|u^{\prime}\right| \leq N$ is not true. Then there exists an interval $\left[t_{1}, t_{2}\right] \subset I$ such that one of the following cases holds:

(i) $u^{\prime}\left(t_{1}\right)=0, u^{\prime}\left(t_{2}\right)=N$ and $0<u^{\prime}(t)<N$ on $\left(t_{1}, t_{2}\right)$,

(ii) $u^{\prime}\left(t_{1}\right)=N, u^{\prime}\left(t_{2}\right)=0$ and $0<u^{\prime}(t)<N$ on $\left(t_{1}, t_{2}\right)$,

(iii) $u^{\prime}\left(t_{1}\right)=0, u^{\prime}\left(t_{2}\right)=-N$ and $-N<u^{\prime}(t)<0$ on $\left(t_{1}, t_{2}\right)$,

(iv) $u^{\prime}\left(t_{1}\right)=-N, u^{\prime}\left(t_{2}\right)=0$ and $-N<u^{\prime}(t)<0$ on $\left(t_{1}, t_{2}\right)$. 
Let us consider the case (i). By (2.1),

$$
\left|u^{\prime \prime}(t)\right|=\left|f^{*}\left(t, u(t), u^{\prime}(t)\right)\right| \leq h(t) g\left(\left|u^{\prime}(t)\right|\right) \quad \text { on }\left[t_{1}, t_{2}\right]
$$

and as a result

$$
\begin{aligned}
\int_{0}^{N} \frac{|u|^{(\sigma-1) / \sigma}}{g(|u|)} d u & =\int_{t_{1}}^{t_{2}} \frac{\left|u^{\prime}(t)\right|^{(\sigma-1) / \sigma} u^{\prime \prime}(t)}{g\left(\left|u^{\prime}(t)\right|\right)} d t \\
& \leq \int_{t_{1}}^{t_{2}} \frac{\left|u^{\prime}(t)\right|^{(\sigma-1) / \sigma}\left|u^{\prime \prime}(t)\right|}{g\left(\left|u^{\prime}(t)\right|\right)} d t \\
& \leq \int_{t_{1}}^{t_{2}} h(t)\left|u^{\prime}(t)\right|^{(\sigma-1) / \sigma} d t \\
& \leq\left(\int_{t_{1}}^{t_{2}}|h(t)|^{\sigma} d t\right)^{1 / \sigma}\left(u\left(t_{2}\right)-u\left(t_{1}\right)\right)^{(\sigma-1) / \sigma} \\
& \leq\|h\|_{\sigma} \varrho^{(\sigma-1) / \sigma} \quad \text { if } 1<\sigma \leq \infty
\end{aligned}
$$

and

$$
\int_{0}^{N} \frac{d u}{g(|u|)}=\int_{t_{1}}^{t_{2}} \frac{u^{\prime \prime}(t)}{g\left(\left|u^{\prime}(t)\right|\right)} d t \leq \int_{t_{1}}^{t_{2}} h(t) d t \leq\|h\|_{1} \quad \text { if } \sigma=1 .
$$

This contradicts (2.2). The other cases are dealt with similarly. This completes the proof of Theorem 1 .

3. Monotone iterative technique. In this section, we develop a monotone iterative technique for our equation, the method being similar to that of [5]. Our conditions are more precise and applicable.

In addition to the hypotheses of the first two sections, we introduce the following hypotheses:

(H2) There exists an $M \in L^{1}(I)$ such that $M(t)>0$ for a.e. $t \in I$ and

$$
f(t, p, s)-f(t, q, s) \geq-M(t)(p-q)
$$

for a.e. $t \in I$ and every $\alpha \leq q \leq p \leq \beta, s \in \mathbb{R}$.

(H3) There exists a $U \in L^{1}(I)$ such that $U(t)>0$ for a.e. $t \in I$ and

$$
f(t, p, s)-f(t, p, y) \geq-U(t)(s-y)
$$

for a.e. $t \in I$ and every $\alpha \leq p \leq \beta, s \geq y, s, y \in \mathbb{R}$.

(H1*) Define

$$
g^{*}(v)=\max \{g(v), \max |\alpha|+\max |\beta|\}, \quad h^{*}(t)=h(t)+2 M(t),
$$


where $g(v)$ and $h(t)$ are as in (H1). Then

$$
\int_{0}^{\infty} \frac{u^{(\sigma-1) / \sigma}}{g^{*}(u)} d u>\varrho^{(\sigma-1) / \sigma}\left\|h^{*}\right\|_{\sigma} .
$$

We have

TheOREm 2. Suppose that $\left(\mathrm{H}^{*}\right)-(\mathrm{H} 3)$ hold. Then there exist monotone sequences $\alpha_{n} \nearrow x$ and $\beta_{n} \searrow z$ as $n \rightarrow \infty$, uniformly on $I$, with $\alpha_{0}=\alpha$ and $\beta_{0}=\beta$. Here, $x$ and $z$ are the minimal and maximal solutions of (1.1) respectively on $[\alpha, \beta]$, that is, if $u \in[\alpha, \beta]$ is a solution of (1.1), then $u \in$ $[x, z]$. Moreover, the sequences $\left\{\alpha_{n}\right\}$ and $\left\{\beta_{n}\right\}$ satisfy $\alpha=\alpha_{0} \leq \ldots \leq \alpha_{n} \leq$ $\beta_{n} \leq \ldots \leq \beta_{0}=\beta$.

Pr o of. For any $q \in[\alpha, \beta] \cap X$, consider the following quasilinear periodic boundary value problem:

$$
\begin{aligned}
& -u^{\prime \prime}(t)=f\left(t, q(t), u^{\prime}(t)\right)+M(t)(q(t)-u(t)), \\
& u(0)=u(2 \pi), \quad u^{\prime}(0)=u^{\prime}(2 \pi) .
\end{aligned}
$$

It is easy to verify that $\alpha$ and $\beta$ are also lower and upper solutions of (3.3) respectively and

$$
\begin{aligned}
\mid f\left(t, q(t), u^{\prime}(t)\right)+M(t) & (q(t)-u(t)) \mid \\
& \leq h(t) g\left(\left|u^{\prime}(t)\right|\right)+2 M(t)(\max |\alpha|+\max |\beta|) \\
& \leq[h(t)+2 M(t)] g^{*}\left(\left|u^{\prime}(t)\right|\right)=h^{*}(t) g^{*}\left(\left|u^{\prime}(t)\right|\right) .
\end{aligned}
$$

Then, by Theorem 1, there exists a solution $u$ of the problem (3.3) with $u \in[\alpha, \beta]$. It is not difficult to show that this solution is unique by using the argument for Lemma 3. Now, define the operator $T: X \rightarrow X$ by $T[q]=u$, where $u$ is the solution of (3.3).

We shall prove:

Claim. If $\alpha \leq q_{1} \leq q_{2} \leq \beta, q_{1}, q_{2} \in X$, then $u_{1}=T\left[q_{1}\right] \leq u_{2}=T\left[q_{2}\right]$.

Indeed, let $y=u_{2}-u_{1}$. Then

$(3.4)-y^{\prime \prime}=f\left(t, q_{2}(t), u_{2}^{\prime}(t)\right)-f\left(t, q_{1}(t), u_{1}^{\prime}(t)\right)+M(t)\left[\left(q_{2}-q_{1}\right)(t)-y(t)\right]$

$$
\geq-U(t) y^{\prime}(t)-M(t) y(t)
$$

Assume that $t_{0}$ is such that $y\left(t_{0}\right)=\min \{y(t): t \in I\}$. We only need to prove that $y\left(t_{0}\right) \geq 0$.

In fact, if $t_{0} \in(0,2 \pi)$ and $y\left(t_{0}\right)<0$, then there would be $0 \leq t_{1}<t_{0}$ $<t_{2} \leq 2 \pi$ such that $y(t)<0$ on $\left(t_{1}, t_{2}\right), y^{\prime}\left(t_{1}\right) \leq 0$ and $y^{\prime}\left(t_{2}\right) \geq 0$. Now (3.4) implies that $y^{\prime \prime}-U(t) y^{\prime}<0$ on $\left(t_{1}, t_{2}\right)$. Solving the differential inequality, we obtain

$$
y^{\prime}\left(t_{2}\right) \exp \left\{-\int_{t_{1}}^{t_{2}} U(t) d t\right\}<y^{\prime}\left(t_{1}\right) \leq 0
$$


which is impossible. If $t_{0}=0$ or $t_{0}=2 \pi$ and $y(0)=y(2 \pi)<0$, then there would be $t_{1}, t_{2} \in(0,2 \pi)$ such that $y^{\prime}\left(t_{1}\right) \geq 0 \geq y^{\prime}\left(t_{2}\right), y^{\prime \prime}(t)-U(t) y^{\prime}<0$ on $\left[0, t_{1}\right) \cap\left(t_{2}, 2 \pi\right]$ and hence

$$
\begin{aligned}
& 0 \leq y^{\prime}\left(t_{1}\right) \exp \left\{-\int_{0}^{t_{1}} U(t) d t\right\}<y^{\prime}(0), \\
& y^{\prime}(2 \pi) \exp \left\{-\int_{t_{2}}^{2 \pi} U(t) d t\right\}<y^{\prime}\left(t_{2}\right) \leq 0,
\end{aligned}
$$

again a contradiction. This proves the claim.

Now, define sequences $\alpha_{0}=\alpha, \alpha_{n}=T\left[\alpha_{n-1}\right], \beta_{0}=\beta$ and $\beta_{n}=T\left[\beta_{n-1}\right]$. Since the solution $u$ of (3.3) satisfies $u \in[\alpha, \beta]$, using the monotonicity of $T$, we see that $\alpha=\alpha_{0} \leq \ldots \leq \alpha_{n} \leq \beta_{n} \leq \ldots \leq \beta_{0}=\beta$. Hence, the $\operatorname{limits}_{\lim _{n \rightarrow \infty}} \alpha_{n}(t)=x(t)$ and $\lim _{n \rightarrow \infty} \beta_{n}(t)=z(t)$ exist. From the previous proof, we know that $\left|\alpha_{n}^{\prime}\right|,\left|\beta_{n}^{\prime}\right| \leq N$ uniformly in $n$. Using the argument for Theorem 1, we know that the sequences $\left\{\alpha_{n}\right\}$ and $\left\{\beta_{n}\right\}$ are equicontinuous and uniformly bounded and hence converge to $x$ and $z$ in $X$. By the definitions, we know that $T[x]=x$ and $T[z]=z$. Then it is obvious by formulas similar to (2.4) and (2.5) that $x$ and $z$ satisfy (1.1).

Furthermore, if $u \in X \cap[\alpha, \beta]$ solves (1.1), then since $T[u]=u$, we have $\alpha_{n} \leq u \leq \beta_{n}$ for any $n=1,2, \ldots$ and hence $u \in[x, z]$ in $I$.

This completes the proof of the theorem.

\section{References}

[1] A. Adje, Sur et sous-solutions généralisées et problèmes aux limites du second ordre, Bull. Soc. Math. Belgique Sér. B 42 (1990), 347-368.

[2] J. Bebernes, A simple alternative problem for finding periodic solutions of second order ordinary differential systems, Proc. Amer. Math. Soc. 42 (1974), 121-127.

[3] A. Cabada and J. J. Nieto, A generalization of the monotone iterative technique for nonlinear second-order periodic boundary value problems, J. Math. Anal. Appl. 151 (1990), 181-189.

[4] J. J. Nieto, Nonlinear second-order periodic boundary value problems with Carathéodory functions, Appl. Anal. 34 (1989), 111-128.

[5] M.-X. Wang, A. Cabada and J. J. Nieto, Monotone method for nonlinear second order periodic boundary value problems with Carathéodory functions, Ann. Polon. Math. 58 (1993), 221-235.

DEPARTMENT OF MATHEMATICS JILIN UNIVERSITY

CHANGCHUN 130023, P.R. CHINA 